Meta

Journal des traducteurs

Translators' Journal

\title{
En équilibre entre deux mondes : un itinéraire de Paris à Jérusalem
}

\section{Francine Kaufmann}

Volume 38, numéro 4, décembre 1993

Le Je du traducteur

The $I$ of the Translator

URI : https://id.erudit.org/iderudit/003289ar

DOI : https://doi.org/10.7202/003289ar

Aller au sommaire du numéro

Éditeur(s)

Les Presses de l'Université de Montréal

ISSN

0026-0452 (imprimé)

Découvrir la revue

Citer cet article

Kaufmann, F. (1993). En équilibre entre deux mondes : un itinéraire de Paris à Jérusalem. Meta, 38(4), 658-663. https://doi.org/10.7202/003289ar d'utilisation que vous pouvez consulter en ligne.

https://apropos.erudit.org/fr/usagers/politique-dutilisation/ 


\title{
EN ÉQUILIBRE ENTRE DEUX MONDES : UN ITINÉRAIRE DE PARIS À JÉRUSALEM
}

\author{
FRANCINE KAUFMANN \\ Université Bar-Ilan, Ramat-Gan, Israël
}

PRÉLUDE

Enfant, je voulais être écrivain! Peut-être aussi maîtresse d'école et comédienne! J'aimais passionnément découvrir les mots et jouer avec les sonorités de la langue française. La première publication d'un de mes poèmes ( $j$ 'avais dix ans) me semblait le signe indubitable que mon destin était tout tracé. J'appris avec étonnement qu'il n'existait pas d'école pour devenir «écrivain». Je fis donc de banales études de lettres... jusqu'à l'inévitable doctorat, nécessaire pour enseigner à l'université. Je me considérais très peu douée pour les langues étrangères. Mes thèmes de latin et d'anglais étaient généralement catastrophiques. Je me rattrapais largement en version. Mais j'avais appris à lire et à écrire l'hébreu dès l'âge de six ans, pour déchiffrer les prières et la Bible. À neuf ans, je rêvais déjà d'aller vivre à Jérusalem. En 1962, l'Éducation nationale admit l'hébreu comme langue vivante au baccalauréat. Je pris des cours particuliers. Je fis mon premier voyage en Israël où je m'essayais maladroitement, passionnément, à parler cette langue ressuscitée que je sentais mienne. Après le bac, parallèlement à mes études de lettres à Nanterre, je m'inscrivis aux Langues Orientales et à une licence d'hébreu dont je passais les épreuves, à l'Université de Lille, le jour même où éclatait la guerre des Six Jours. Je m'estimais toujours peu douée pour les langues. L'hébreu était ma patrie, non un idiome étranger. Je trouvais néanmoins ironique d'obtenir mon premier poste d'assistante à la Sorbonne Nouvelle, non pas en littérature française mais en troisième année de licence d'hébreu. En plus de l'histoire de la langue et de la littérature, $\mathrm{j}$ 'enseignais la version-thème, mon premier contact avec la «traduction» à un niveau qui dépassait les fades exercices scolaires. J'avais 22 ans et je considérais toujours la traduction comme une discipline «auxiliaire». Je n'aurais jamais imaginé en faire une profession. J'avais pourtant un oncle à Genève qui dirigeait la section française de traduction à l'ONU. Mais je voyais en lui un haut fonctionnaire et ne songeais pas à l'interroger sur ses activités.

À cette époque j'entamais une carrière parallèle (que je n'ai jamais abandonnée) de journaliste dans la presse juive et d'assistante, puis de réalisatrice, à la radio et à la télévision. Pourtant mes vieux démons littéraires m'habitaient toujours. Je commençais à traduire et à publier des poèmes hébraïques, m'essayais à traduire des nouvelles. Je me souviens d'une nuit où, réveillée par un rythme longtemps recherché, je me levai et traduisis en quelques heures, en vers et en alexandrins, une longue ode du poète de la renaissance nationale hébraïque, Bialik. La fièvre et l'exaltation de la création m'animaient.

Je reçus avec surprise la proposition de la très sérieuse Documentation catholique de traduire une interview de Golda Meïr accordée au quotidien israélien Maariv, après son entrevue avec le Pape, en 1973. Ma première traduction non littéraire!

Le texte parut avec les commentaires violemment critiques et anti-israéliens de la rédaction et fut repris par de nombreux organes de presse francophones qui voyaient là un document significatif des relations entre Israël et le Vatican. J'avais le sentiment d'avoir été piégée. On avait utilisé ma connaissance de l'hébreu à des fins hostiles! Je retrouvais récemment cette impression lorsque, la Croix Rouge Internationale s'étant adressée à un 
bureau de Genève pour obtenir la traduction d'une brochure de Tsahal (l'armée israélienne), le texte aboutit chez moi. Mais cette fois je comprenais l'enjeu. Je connaissais les positions ouvertement anti-israéliennes de la CRI, qui se refuse toujours à admettre le Magen David Adom dans ses rangs. Heureusement la brochure se contentait d'expliquer aux officiers l'importance humanitaire de la Croix Rouge et recommandait d'être courtois. Je pouvais donc traduire aussi fidèlement que possible, sans me sentir espion ou «agent double». Mais je pesais néanmoins soigneusement le choix des synonymes.

\section{LES DÉBUTS}

En apparence, c'est le hasard qui a fait de moi une traductrice et une interprète. En apparence seulement. Le jour où je fis le saut pour quitter mon Paris natal et m'installer en Israël, je n'avais toujours trouvé aucun poste en littérature, dans une université israélienne. La chance voulut que le professeur Neher (qui m'avait conseillée durant ma thèse de doctorat) connaisse un voisin qui recherchait un professeur de traduction pour l'Université Bar-Ilan à Ramat-Gan. Il s'agissait de mettre sur pied une section française, dans la nouvelle école de traducteurs et d'interprètes qui y avait été créée, deux ans auparavant, en 1972. Ma formation de chercheur, d'enseignante et de journaliste me permit d'obtenir le poste. Avant de quitter l'Europe, je fis le tour des bureaux de l'ONU à Genève et de l'UNESCO à Paris, rencontrai des traducteurs salariés et indépendants, des enseignants des écoles de traduction. J'amassai de la documentation, des notes, dévorai en bibliothèque tous les livres que je trouvais sur la question. Bien entendu, il n'existait pratiquement rien dans le domaine hébreu-français. Mais il était exaltant, précisément, de partir dans un pays neuf pour y faire quelque chose d'inédit.

Je devins traductrice professionelle parce que j'enseignais la traduction. Je ne pouvais décemment pas former des étudiants sans connaître moi-même le marché du travail. J'aimais aussitôt cette nouvelle activité que je sentais très proche de ma pratique journalistique. Après tout, il s'agissait d'assimiler un sujet nouveau, comme dans un reportage, de se pénétrer de la pensée et de la personnalité de l'auteur d'un texte (comme dans une interview) et de réexprimer dans un français aussi clair et précis que possible ce que je venais moi-même de comprendre, en m'adressant à des lecteurs aussi ciblés que les lecteurs d'un journal donné. Sans compter le plaisir de trouver le mot juste, de jongler avec les idées et les styles.

Souvent je bénissais la chance qui m'avait coupée du monde imaginaire de la littérature pour me plonger avec force dans l'actualité et dans les contingences pratiques et techniques de la réalité. Je passais avec délice de l'agriculture à l'économie, de la numismatique à la psychanalyse. Mais je continuais, pour mon plaisir, à traduire mes chers poèmes... dont je nourrissais mes programmes de radio, rêvant d'une anthologie (que j'ai d'ailleurs publiée par la suite, dans une édition, hélas, confidentielle).

Peu à peu, mes collègues interprètes de l'Université Bar-Ilan me suggérèrent d'essayer l'interprétation simultanée. En Israël, un nombre appréciable d'interprètes de «l'époque hérö̈que» venaient de la radio: la pratique du micro, du direct, l'habitude du reportage leur permettaient d'égaler rapidement les interprètes formés dans les écoles de Genève, de Buenos Aires ou d'ailleurs.

Je refusais tout net! J'ignorais tout de la profession et le fait même d'écouter tout en parlant, sans se laisser distraire des heures durant, me semblait relever de la pure magie.

Là, encore, le hasard me fit changer d'avis. Au moment de la visite surprise du Président Sadate à Jérusalem, en novembre 1977, une partie des interprètes français étaient soit à l'étranger, soit journalistes, réquisitionnés par leur rédaction. En visitant, en voisine, le centre de presse qu'on montait à la hâte au Theâtre de Jérusalem, je rencontrais un collègue interprète de Bar-Ilan, affolé de ne pouvoir compléter son équipe. Il me planta 
devant un écran de télévision, me fit répéter simultanément durant plus de dix minutes un dialogue stupide, en argot américain, du film de gangsters alors diffusé, décréta que j'étais une interprète-née et m'engagea sur-le-champ ! Faute de mieux! Le surlendemain, terrorisée et survoltée, je fis mes débuts devant mes collègues, scandalisés et médusés qu'on m'ait engagée dans de telles circonstances... et que j'aie osé accepter. Ils avaient bien sûr raison. Mais j'avais l'impression d'agir dans un rêve.

Pour les discours en hébreu, tout se passa relativement bien. Puis vint la conférence de presse en anglais de Begin et de Sadate, assis à quelques mètres de nous sur la scène du Théâtre. Et moi qui, malgré mes dix ans d'anglais scolaire, me considérais toujours comme une parfaite cancre en langues, je m'entendais traduire ces paroles qui, à Jérusalem, même dans leurs habits anglais, se donnaient sans peine des allures de prophéties bibliques: «No more war, no more bloodshed! Peace is the word...»

Il va sans dire que je ne souhaite à personne de faire ses débuts dans de telles circonstances. Mais la nécessité produit parfois des miracles. Désormais l'interprétation ne me faisait plus peur. Je demandais à mes collègues de la section anglaise de me laisser suivre leurs cours, $\mathrm{j}$ 'assistais à tous les congrès qui passaient à ma portée, assise au dernier rang en marmonnant à mi-voix : mes voisins me croyaient folle. J'entrepris des voyages linguistiques en Angleterre et aux États-Unis. Je me perfectionnais.

\section{LA PASSION}

Avec le temps, je suis devenue une véritable interprète, membre de l'AIIC, et j'ai le bonheur et la fierté de retrouver en cabine des collègues qui furent mes étudiants en interprétation, à Bar-Ilan. Je traduis beaucoup moins par écrit; même si je continue d'enseigner la traduction écrite, je m'efforce d'accepter, aussi souvent que possible, des travaux limités et variés.

Mais c'est à ma profession d'interprète que je dois mes grands moments d'émotion. Entre deux cours, deux émissions, deux articles ou deux congrès sur la plomberie, le sionisme, les énergies nouvelles, le sport, l'irrigation, le droit, les régimes de sécurité sociale, la gestion de la charge électrique, la métallurgie, le racisme ou la graphologie... je vis des instants exaltants où l'Histoire s'accomplit sous mes yeux. Alors mon identité juive et mon identité française s'épousent et se fécondent, je deviens une petite pierre dans l'entreprise de reconstruction de l'État d'Israël, j'assiste en privilégiée à la réalisation du rêve des prophètes.

Je ne suis pas encore devenue écrivain (pas même un vrai poète), mais c'était bien moi cette silhouette aux côtés des présidents Navon et Mitterand, lors de la première visite officielle en Israël d'un chef d'Etat français, en mars 1982. Chaque fois que les deux hymnes nationaux retentissaient, je sentais bien que la «double allégeance» n'existe pas, que ma double identité constitue ma richesse et ma fierté et me permet d'être un petit pont jeté entre mes deux patries, maniant des mots pour mieux unir des idées, des manières différentes et pourtant si semblables d'être homme ou femme de ce temps.

C'est moi encore cette voix étranglée qui résonne et dit «je» à la fin du film Shoah. Lorsque, en 1979, Claude Lanzmann m'a choisie, moi, fille de déporté, docteur en littérature de la Shoah, pour interpréter, dans son film, à partir de l'hébreu, les témoignages recueillis en Israël, je n'ai pas réussi à le convaincre qu'il me demandait de déroger à toutes les règles de ma profession. Mais convaincue de la grandeur de son entreprise, j'ai accepté de mémoriser, sans prendre de notes, des récits insoutenables, émaillés de chiffres et de noms, sans interrompre les témoins qui revivaient leur passé, les yeux fixés dans les miens, tandis que, coincée près de l'objectif pour que le regard de notre interlocuteur se noie dans l'wil de la caméra, j'étais tenue de restituer l'horreur qui s'enregistrait, par bobines de dix minutes sur l'impitoyable bande sonore qu'on ne pourrait plus retoucher par la suite. 
C'est moi toujours qui danse la Hora dans un hôtel de Berlin, en 1988, juive bien vivante échappée aux griffes nazies, ayant posé pour la première fois le pied sur le sol allemand afin de participer, avec une équipe d'interprètes israéliens, à un congrès sur l'éthique médicale juive.

C'est moi encore, assise dans un studio de la radio, à Paris, durant mon année sabbatique, traduisant en direct pour les auditeurs des radios juives de France les bulletins d'information de la radio israélienne durant la guerre du Golfe. Ces émissions spéciales, qui devaient durer quatre jours - lors de l'ultimatum du 15 janvier 1991, se prolongèrent durant deux mois, avec des alertes de nuit où nous restions branchés sur Israël, par le miracle du téléphone. La ligne s'évanouissait par instants ou se brouillait (ce qui m'a fait renoncer, dès le premier jour, à l'interprétation simultanée au profit de la consécutive). Mais, la plupart du temps, nous vivions en direct chaque seconde de l'angoisse montante. Je me souviens notamment de la première alerte sur Tel Aviv. Notre correspondant dut s'interrompre pour s'enfermer, avec sa famille, dans sa chambre hermétique, bouclant à la hâte les horribles masques à gaz. À ma demande, il avait posé le combiné près du poste ouvert de radio qui égrenait les instructions de sécurité, quand soudain il interrompit ma traduction pour me demander, la voix déformée par le masque, ce qu'il devait faire à présent. Dans la bousculade il avait mal entendu le speaker et, de Paris, nous lui avons précisé les directives de la radio. Le lendemain nous avons interviewé, grâce à son téléphone de voiture, le ministre israélien de la santé, en route vers Jérusalem pour mettre au point les mesures à prendre en cas d'attaque chimique. Et, au fil des jours, bien des personnalités israéliennes de premier plan ont pu intervenir par téléphone (la barrière linguistique étant levée grâce à l'interprétation consécutive) sur les ondes de Radio-Communauté. En février, durant huit jours c'est d'Israël que j'ai diffusé vers la France les nouvelles de Kol Israël retransmises de mon poste de radio, traduisant et commentant au gré des alertes, décrivant les destructions massives causées par la chute d'un seul missile Scud à quelques centaines de mètres de l'appartement de mon frère, chez qui j'avais passé la nuit. Parallèlement, j'interprétais, lors d'un congrès organisé à Jérusalem par l'ORT, qui marquait ainsi sa solidarité avec l'État menacé, et je recueillais, chez I'écrivain David Grossman et l'historien Zeev Sternhell, des textes écrits à chaud que je traduisais (par écrit) pour le journal Libération, à Paris. Par ailleurs, pendant toute la durée de la guerre, Libération commanda à Ze'ev Schiff, commentateur militaire du quotidien israélien Haaretz, des articles sur les perspectives stratégiques vues d'Israël. Ces jours-là, je courais au journal et nous appelions Ze'ev Schiff qui dictait son analyse au téléphone. Je disposais d'une heure ou deux pour traduire, mettre en forme et corriger, avec le réviseur, l'article qui partait aussitôt à l'imprimerie. Par la suite, Ze'ev Schiff s'excusa de nous avoir livré, plus d'une fois, un texte passablement improvisé et décousu, parce qu'il se trouvait alors en pleine alerte, enfermé dans sa chambre hermétique. Ma «guerre du Golfe» s'acheva le jour où je traduisis en direct (la nouvelle n'était pas encore tombée sur les téléscripteurs des agences de presse) l'ordre du porte-parole de l'armée israélienne de rouvrir les chambres hermétiques et de relâcher la vigilance. Grâce à mon métier, j'avais eu la chance de ne pas rester, angoissée, passivement à l'écoute de mon pays attaqué, alors que je me trouvais en année sabbatique à Paris.

Quelques mois plus tard, une première boucle, entamée pour moi avec la visite à Jérusalem du président Sadate, se refermait. Je reçus un coup de téléphone de Washington, des services d'interprétation du secrétariat d'État, m'invitant à partir quelques jours plus tard à Madrid pour assurer l'interprétation en français lors de la Conférence de paix. Je crus d'abord à une plaisanterie de mauvais goût et gardai le secret durant 24 heures. Mais un second appel, de Madrid, me convainquit bientôt. Journées inoubliables où, assise dans ma cabine, à un mètre de la délégation libanaise, et à trois mètres des délégués israéliens, 
je récitais pour moi seule, juste avant l'ouverture, des prières en hébreu pour la paix. En coulisse, notre petite communauté d'interprètes vivait déjà concrètement les prémisses de l'harmonie et de la coopération internationales. Nous étions en effet quatre Israéliennes, cinq Arabes, des Russes et des Américains, collègues vite devenus amis, soudés, pour donner le meilleur de nous-mêmes, afin de permettre une vraie communication verbale, premier stade d'une communication des esprits. Il y eut d'ailleurs des moments surréalistes où moi, Israélienne, je dus traduire pour mes «clients» francophones, ignorants de ma situation schizophrénique, les discours enflammés de ceux qui sont encore nos ennemis d'aujourd'hui. Grâce aux enregistrements réalisés à mon insu par les radios juives de France, j'ai pu constater que ma voix n'avait pas tremblé, que j'avais même honnêtement restitué les émotions et la conviction de ceux qui disaient par ma voix : «Nous, les Palestiniens sous la botte israélienne... nous, les Syriens face à l'adversaire sioniste....» Qui pourrait deviner qu'aussitôt mon rôle terminé j'étais sortie de cabine pour éclater en sanglots nerveux et silencieux après avoir fidèlement reproduit les imprécations du ministre syrien des Affaires étrangères?

Il est vrai que quiconque se veut interprète doit être aussi un peu acteur, apprendre à s'oublier pour devenir autre et se couler dans la peau du personnage qu'il est chargé d'incarner, même lorsque ce dernier est radicalement différent de soi. Je me souviens d'ailleurs avec excitation de ce jour où, venue d'Israël avec dans mes valises les bobines d'un film à peine sorti de la table de montage, je m'assis dans une salle de projection des Champs-Élysées, à Paris, derrière les membres du jury du Festival de Cannes pour leur chuchoter à l'oreille les dialogues où j'interprétais tous les rôles, avec la conviction sincère d'une comédienne frustrée.

\section{OMBRES ET LUMIÈRES}

Grâce à ma profession, j'ai côtoyé des figures légendaires que je n'aurais jamais rêvé approcher (dont Golda Meïr et Moshé Dayan). Des orateurs accomplis (Mena'hem Begin, Abba Eban, Joseph Burg) m'ont galamment affirmé que ma traduction sonnait mieux que l'original! Shlomo Hillel (alors président de la Knesset, qui comprend et parle le français) a estimé, satisfait en m'écoutant, que son discours avait été bon.

Certes, il y a aussi des moments sans lustre, mais où le cœur se dilate parce qu'une salle entière rit ou s'émeut au même instant (avec un décalage imperceptible dû à l'interprétation), où quelqu'un cite l'intervenant précédent en reprenant les termes entendus dans son casque, où l'interprète corrige adroitement, sans y toucher, une bourde de l'orateur ou du président de la. séance, adapte pour son public, en une fraction de seconde, une remarque inconcevable en français. Quand tout va bien et que personne ne se plaint (les médecins sont alors convaincus qu'il est lui-même médecin et les juristes le croient juriste), l'interprète est souvent le seul à être conscient que, si la communication passe dans la salle, c'est par son intermédiaire. Certains le croient d'ailleurs superflu: «Mais ma pauvre, il ne fallait pas vous fatiguer à traduire, nous avions des appareils avec les discours en français !», m'a dit ingénument une congressiste. Un appariteur a refusé l'entrée à une collègue qu'il prenait pour une resquilleuse : «Il y a déjà des appareils de traduction, a-t-il déclaré péremptoire. Pas besoin d'interprètes !»

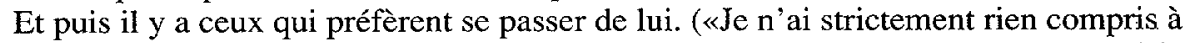
tous ces débats» m'a confié une vieille dame. «C'était horriblement technique» ai-je renchéri, compatissante. «Je ne sais pas. Je ne comprends ni l'hébreu ni l'anglais.» «Mais pourquoi n'avez-vous pas eu recours à l'interprétation?» «Ah non, il faut supporter ces horribles écouteurs.») D'autres congressistes, lorsqu'ils s'ennuient, s'amusent à passer d'une chaîne à l'autre, comparent et notent les interprètes, soudain hilares s'ils prennent 
en défaut le malheureux qui décidément ne comprend rien au rôle des inhibiteurs calciques en cardiologie.

Car il y a, bien sûr, les moments de déroute où l'on bafouille lamentablement, perdu dans de longues démonstrations scientifiques que l'orateur lit au galop, en avalant ses mots pour terminer sa communication dans le «temps trop bref qui lui est imparti». Sans compter les moments où l'on retient son fou rire pour restituer sans sourciller certaines interventions balbutiées dans un anglais difficilement reconnaissable, pendant que la salle, qui écoute l'original, se dévisage en faisant poliment semblant de comprendre.

Et quelle belle séquence pour un film comique que cet instantané où, debout à l'aéroport près de l'avion présidentiel, j'essayais de faire bonne figure, trempée comme une soupe parce que le chef du protocole m'avait «emprunté» à la hâte, pour protéger Madame Mitterand, mon beau parapluie doublé et à volants (que je n'ai jamais revu depuis), tandis que stylo et crayon se refusaient à fonctionner sur mon papier ruisselant et que l'interprète venu de France me repoussait littéralement loin du micro, ayant décrété que l'interprétation française serait assurée par lui seul. Et devant l'œil bonasse de la caméra des Actualités, il mima avec conviction une prise de notes consécutive avant de lire - à ma place - le texte du discours qu'il m'avait déjà pris des mains et que j'avais laborieusement traduit mot à mot avec le Président Navon. (Les jours suivants, «mon» président eut parfois recours à son aide de camp pour écarter, au moment opportun, celui qu'il n'appela plus que : «habiriyone», qu'on peut traduire librement par: le goujat.)

Heureusement pour l'honneur de la profession, ces petites guerres-là ne font pas la une des journaux. Mais il est vrai qu'ils n'ont pas toujours le caractère facile, ces interprètes enfermés, des jours durant, dans des cabines exiguës à l'air confiné, donnant sur des salles sans fenêtres, soumis à des horaires inégaux et aux caprices d'employeurs, dont certains s'obstinent encore à les traiter comme du personnel subalterne ou les assimilent à de simples machines, capables de toutes les performances. Obligé par ailleurs de défendre chèrement sa place au soleil dans un marché encombré et trop restreint, l'interprète doit donc être et rester toujours le meilleur. C'est particulièrement vrai en Israël, pays où ne siège aucune organisation internationale, où beaucoup de congrès se déroulent uniquement en anglais, pays lilliputien car, si l'on exclut le désert, la population juive de quatre millions d'habitants vit concentrée sur moins de 10000 kilomètres carrés.

On se surprend donc à rêver d'un Moyen-Orient ouvert et uni, où l'interprète israélien, aujourd'hui claustrophobe, pourrait traverser une frontière voisine non hostile et travailler avec ses collègues du Caire et de Damas, où l'École d'interprètes de Bar-Ilan échangerait des professeurs et des étudiants avec l'École d'interprètes d'Amman. À l'époque de l'âge d'or espagnol, les traducteurs juifs et arabes ne servirent-ils pas tous deux de pont entre l'Orient et l'Occident? Ben-Gourion affirmait qu'en Israël celui qui ne croit pas aux miracles n'est pas réaliste. À l'heure où Bar-Ilan s'apprête à former une première promotion de traducteurs et d'interprètes, pour le marché local, dont la combinaison comporte le russe (chose impensable il y a quelques années), il ne semble pas imprudent d'affirmer que, d'ici l'an 2000, de nombreux congrès exigeront la combinaison hébreu-arabe qui deviendra alors naturelle... et rentable.

Le traducteur et l'interprète ne sont-ils pas par essence des intermédiaires privilégiés entre les peuples et les cultures? Alors: métier ancilliaire? Perroquet d'un autre qui pense, écrit et parle à sa place? Allons donc !

Consciente des richesses de chaque particularisme, mais soucieuse de mieux servir l'universel, l'ouverture et la tolérance, la confrérie des traducteurs est l'instrument irremplaçable de la communication planétaire. 